\title{
On Decreasing Utilization: Models of Care for Frequently Hospitalized Patients and Their Effect on Outcomes
}

\author{
Paul D. Hain, MD*, Bharath Thankavel, MD, Leanne Metcalfe, PhD
}

Blue Cross Blue Shield of Texas, Richardson, Texas.

$\mathrm{n}$ this month's edition of the Journal of Hospital Medicine, Goodwin and colleagues report their findings from their systematic review of models of care for frequently hospitalized patients. The authors reviewed the literature for interventions to reduce hospital admissions in frequently hospitalized patients with the goal of assessing the success of the interventions. This report contributes to the literature base of interventions to reduce healthcare utilization, particularly in the area of inpatient hospitalization. ${ }^{1}$

Goodwin et al. report that only nine studies met their criteria for review after a thorough search of the published literature. Of these nine studies, only four were determined to be high-quality studies. Interestingly, the low-quality studies found positive results in reducing hospital utilization, whereas the high-quality studies found decreases that were mirrored by their control groups. Impressive heterogeneity was found in the range of definitions, interventions, and outcome measures in the studies. These studies highlight the issue of "regression to the mean" for sicker individuals; however, they may not address readmission rates of specific medical systems or procedures that are also cost drivers, even if the patients are not considered critically ill. They also show where research partnerships can assist in increasing the number of members included in the studies for robust analyses.

From the perspective of a health plan, we applaud all efforts to improve patient outcomes and reduce cost. This report states that efforts to reduce chronic hospitalizations have not been unqualified successes. We must reflect upon how reducing utilization and improving outcomes align with our overall goals as a society. Recently, Federal Reserve Chairman Jay Powell summed up our nation's particular issue, stating, "It is widely understood that the United States is on an unsustainable fiscal path, largely due to the interaction between an aging population and a healthcare system that delivers pretty average healthcare at a cost that is much higher than that of any other advanced economy." 2

A recent Kaiser Family Foundation analysis showed that $1 \%$ of patients accounted for $23 \%$ of all medical spending in the United States, and $97 \%$ of medical spending is attributed to

*Address for correspondence: Paul D. Hain, MD, Blue Cross Blue Shield of Texas, 1001 East Lookout Drive, Richardson, Texas 75082; Telephone: 972-7667032; Fax: 972-766-6008; Email: Paul_Hain@BCBSTX.com

Published online first Octobr 31, 2018.

Received: August 12, 2018; Accepted: August 17, 2018

( 2018 Society of Hospital Medicine DOI 10.12788/jhm.3089 the top $50 \%$ of patients. ${ }^{3}$ Pharmaceutical costs also play a role in this trend. Blue Cross and Blue Shield of Texas (BCBSTX) found that $2.5 \%$ of our population accounted for just under $50 \%$ of total medical spending. Conversely, when looking at patients with very high costs, only $0.4 \%$ had over $\$ 100,000$ in spending exclusive of pharmacy. When including pharmacy, that number rises to $0.5 \%$. As we consider annual medical and pharmacy trends year over year, we find that pharmacy spending may outpace hospital expenses in the near future.

Our internal data are also consistent with published reports that fewer than half of high-cost patients in one year continue to be high-cost cases the following year. Niall Brennan et al. reported that only $39 \%$ of the top $5 \%$ of spenders in a given year are also high spenders the following year. ${ }^{4}$ This finding not only coincides with the author's statement around regression to the mean for the high admission utilizers, but it may be instructive to those looking to a Pareto method of attacking cost. If more than half of targeted patients will move out of the high cost category on their own, then demonstrating the effectiveness of interventions becomes challenging. Moreover, this regression finding speaks to the need to create effective programs to manage population health on a broad basis, which can address quality to all members and streamline costs for a large group that covers well more than $50 \%$ of medical spending.

BCBSTX emphasizes the creation of systems that let providers become responsible and accountable to outcomes and cost. Accountable Care Organizations (ACOs) and Intensive Medical Homes (IMHs) have played important roles in this journey, but physicians need to continue to invent and prioritize interventions that may achieve both goals. In particular, hospitalists have an important role to play. As ACOs flourish, hospitalists will need to join under the value-based umbrella and continue to intervene in patient care, policies, and procedures to reduce avoidable hospitalizations.

The development of value-based arrangements offers the healthcare system a unique opportunity to bring much-needed change. In our medical partnerships, direct communication with providers regarding their member experience and sharing of vital information about their patients' health status have helped improve patient outcomes and decrease cost. Our IMH partnerships show a savings of up to $\$ 45,000$ per member per year driven by decreases in admissions and ER visits, and in some cases, expensive medications. The hard work in these successes lies within the subtleties of fostering the relationship between payers and providers. Each pillar within the ecosystem plays a key role offering strengths, but the upside toward 
change comes in how we support each other's weaknesses. This support is manifested in two ways: collaboration through communication and transparency through data sharing.

The road to change is one less traveled but not unpaved; advances in technology allow us to take experiences and build from them. At its core, technology has enhanced our collaboration and data capabilities. The ability to stay in touch with providers allows for almost real-time addressing of issues, promoting efficiency. The connection we have with providers has evolved from being solely paper contracts to a multichannel, multifunctional system. The ability to take claims experience, insert clinical acumen, and perform data analysis brings actionable solutions to be executed by our providers.

Those in the healthcare system will need to come together to continue to create interventions that improve quality while decreasing costs. The second part may require even more work than the first. The Health Care Cost Institute recently published data showing that inpatient utilization over a five-year period fell $12.9 \%$ in the commercially insured. ${ }^{5}$ However, over that same period, hospital prices for inpatient care rose $24.3 \%$. The fundamental reason for the excess amount of money spent in United States healthcare is that the prices are incredibly high. ${ }^{6}$ Currently, when diligence is exercised in reducing utilization, hospitals simply raise prices as a response to compensate for the lost income. Likewise, although prescription drug utilization only increased $1.8 \%$ during that period, the prices increased by $24.9 \%$.
For the US healthcare system to improve its quality and reduce its cost, we will need inventive partnerships to continue to create new systems to interact with patients in the most efficient and effective way possible. Readmissions and hospital utilization will be a large part of that improvement. Hospitals and hospitalists should ensure that they continue to focus on making healthcare more affordable by improving efficiency and outcomes and by resisting the tendencies of hospitals and pharmaceutical companies to raise prices in reaction to the improved efficiency.

Disclosures: The authors have nothing to disclose.

\section{References}

1. Goodwin A, Henschen BL, O'Dwyer LC, Nichols N, O'Leary KJ. Interventions for Frequently Hospitalized Patients and their Effect on Outcomes: A Systematic Review. J Hosp Med. 2018;13(12):853-859. doi: 10.12788/jhm.3090.

2. Marketplace. Fed Chair Jay Powel. https://www.marketplace.org/2018/07/12/ economy/powell-transcript. Accessed August 3, 2018.

3. Health System Tracker. https://www.healthsystemtracker.org/chart-collection/health-expenditures-vary-across-population/\#item-start\%20 12/01/2017. Accessed August 3, 2018.

4. NEJM Catalyst. Consistently High Turnover in the Group of Top Health Care Spenders. https://catalyst.nejm.org/high-turnover-top-health-care-spenders/. Accessed August 3, 2018.

5. Health Care Cost Institute. 2016 Health Care Cost and Utilization Report. http://www.healthcostinstitute.org/report/2016-health-care-cost-utilization-report/. Accessed August 3, 2018.

6. Anderson GF, Reinhardt UE, Hussey PS, Peterosyan V. It's the prices, stupid: why the United States is so different from other countries. Health Aff (Millwood). 2003;22(3):89-105. doi: 10.1377/hlthaff.22.3.89. 